\title{
Mathematical Modeling of Pressure on Cylindrical Ellipse using Side-by-Side Configuration
}

\author{
Chairul Imron and Mahmud Yunus
}

\begin{abstract}
The application of the concept of fluid is often used to solve problems in the daily life. One of them is the problem of fluid around an elliptical cylinder. This study aims to solve the problems of the fluid around two elliptical cylinder configuration with side-by-side using the Navier-Stokes equations. Navier-Stokes equations-incompressible, viscous and unsteadyare solved using finite difference method staggered grid and SIMPLE (Semi Implicit Method for Pressure-Linked Equation) algorithms. Finite difference method is used to complete the grid arrangement, whereas the SIMPLE algorithm is used to obtain components of velocity and pressure value. Results of this study are the pressure value based on fluid flow profile and a mathematical model which received an elliptical cylinder pressure. Profile of fluid flow is simulated by varying the Reynolds number of 100, 1000, 7000, and 10000 as well as variations in the distance between the cylinder with a ratio of $2 \leq S / a \leq 6$ where $L$ is the distance between the cylinder and $a$ is the minor axis of the cylinder ellipse. Then the pressure is calculated based on the value of the received cylinder pressure components. After obtaining the pressure value, then we create a mathematical model of the stresses imposed on the elliptical cylinder.
\end{abstract}

Index Terms-Drag coefficient, Navier-Stokes equations, passive control.

\section{INTRODUCTION}

$\mathbf{M}$ INIMIZING the drag accepted by body of the car is needed to obtain a suitable car body design, especially for racing cars. This technique is also used in catamaran boat design. The basic concept for analyzing the drag is also used in a variety of shapes ranging from boxes, cylinders, circular, elliptical and other shapes modifications. Then it is developed with the object of more than one configuration with tandem or side-by-side.

Singha and Sinhamahapatra conduct research fluid flow passing through the cylindrical single circular object with Reynolds numbers at $45 \leq R_{e} \leq 250$ and perform experiments on the distance between the dividing wall which is $2 \leq H / D \leq 8$ [1]. Sarvghad-Moghaddam and Nooreddin conducted a study of fluid flow around two circular cylinders arranged side-by-side with the Reynolds number 100 and 200 for a laminar flow, $10^{4}$ to turbulent flow. Then the position within the cylinder using a ratio that varies at $2 \leq L / D \leq 4$ is $1.5,3,4$ [2].

This paper discusses a two-dimensional fluid flow around two elliptical cylinders arranged side-by-side to determine the pressure received at four different points with attention to fluid flow and pressure profiles received by both cylinder ellipses.

Manuscript received January 11, 2016; accepted January 20, 2016.

The authors are with the Department of Mathematics, Institut Teknologi Sepuluh Nopember, Surabaya 60111, Indonesia. Emails: \{imron-its, myunus\} @matematika.its.ac.id

This work has been supported by the research centre of Institut Teknologi Sepuluh Nopember 063177.02/IT2.11/PN.08/2015.

\section{NUMERICAL METHOD}

The equations used for unsteady, viscous and incompressible fluid is the Navier-Stokes equations

$$
\begin{aligned}
& \frac{\partial \mathbf{u}}{\partial t}+\nabla \cdot \mathbf{u u}=-\nabla P+\frac{1}{R_{e}} \nabla^{2} \mathbf{u} \\
& \nabla \cdot \mathbf{u}=0 .
\end{aligned}
$$

where $\mathbf{u}$ is the velocity, $R_{e}$ is the Reynolds number, and $P$ is the pressure. Navier-Stokes equations above can be solved numerically using the SIMPLE algorithm. The algorithm consists of several steps. The first step is to provide an initial value for each variable, and then completed the momentum equations to obtain the velocity components ignoring initial pressure components, so that (1) becomes

$$
\frac{\partial \mathbf{u}}{\partial t}=-\nabla \cdot \mathbf{u u}+\frac{1}{R e} \nabla^{2} \mathbf{u}
$$

the right-hand side is solved using finite difference, and the second step is carried out

$$
\frac{\partial \mathbf{u}}{\partial t}=\frac{\mathbf{u}^{* *}-\mathbf{u}^{*}}{\triangle t}=-\nabla P
$$

We now put divergence on both sides, and the result is

$$
\frac{\nabla \mathbf{u}^{* *}-\nabla \mathbf{u}^{*}}{\Delta t}=-\Delta P
$$

by considering (2), we obtain $\nabla \mathbf{u}^{* *}=0$. It follows that the equation turns into

$$
\frac{\nabla \cdot \mathbf{u}^{*}}{\triangle t}=-\Delta P
$$

Equation (6) is called Poisson's equation. Completion of the Poisson equation solution will more quickly to achieve convergence, if SOR (Successive Over Relaxation) is used, as follows

$$
\left(P_{n}\right)_{i, j}=(1-\omega)\left(\left(P_{n-1}\right)_{i, j}\right)+\omega\left(P_{n}\right)_{i, j}
$$

This equation will yield a value of $P$. The final step is the correction of velocity equation

$$
\frac{\partial \mathbf{u}}{\partial t}=-\nabla P
$$




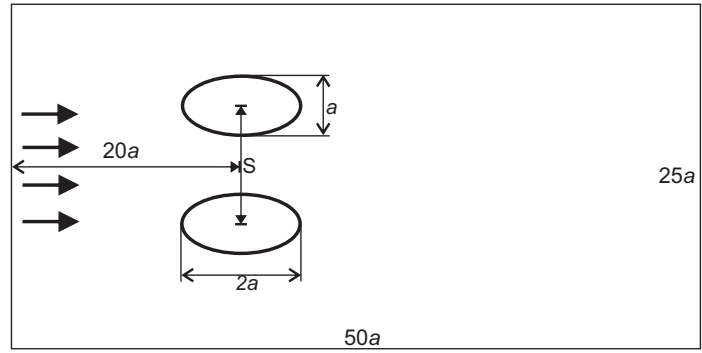

Fig. 1. Scheme used in this research.

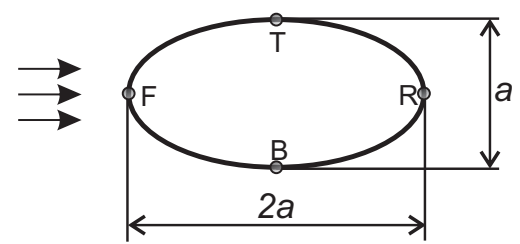

Fig. 2. Pressure scheme at ellipse.

TABLE I

COMPARISON OF DRAG COEFFICIENT OF A CYLINDER FOR $R_{e}=100$

\begin{tabular}{c|cccc}
\hline Researcher & Imron [3] & Zulh [4] & Lima [5] & Pan [4] \\
\hline$C_{D}$ & 1.358 & 1.4 & 1.39 & 1.32 \\
\hline
\end{tabular}

\section{Main Results}

We simulate the Navier-Stokes equations by using a numerical method. The space of interest is partitioned into $25 a \times 50 a$ grids, where $a$ is length of minor axis of ellipse cylinder and $S$ is the distance between the cylinder. Two cylindrical ellipses using side-by-side configuration are placed at distance $20 a$ from the front side. The value of $S / 2 a$ is varied between 1.0, 2.0 and 3.0, as shown in Fig. 1.

The results of a simulation program is accurate, if the simulation results are closer to the experimental value. Drag coefficient of the experimental results compared with simulation results can be seen in Table I.

TABLE II

DRAG COEFFICIENT $C_{D}$ ON $R_{e}$ WITH $S / D=1.0,2.0$ AND 3.0

\begin{tabular}{c|c|llll}
\hline & $R_{e}$ & 100 & 1000 & 7000 & 10000 \\
$S / 2 a$ & $\ln R_{e}$ & 4.605 & 6.908 & 8.854 & 9.210 \\
\hline 1.0 & $C_{D}$ & 1.0843 & 1.0065 & 1.4472 & 1.4991 \\
2.0 & $C_{D}$ & 0.9937 & 0.8935 & 1.3457 & 1.3734 \\
3.0 & $C_{D}$ & 1.0066 & 0.9379 & 1.3595 & 1.3697 \\
\hline
\end{tabular}

The result of the simulation program with $R_{e}=$ $100,1000,7000$, and 10000 and $S / 2 a=1.0,2.0$, and 3.0, can be seen in Table II, and the graphs can be seen in Fig. 3 .

Twelve drag coefficient data for $R_{e}=100,1000,7000$, and 10000 and $S / 2 a=1.0,2.0$, and 3.0 in Table II was obtained using the following three equations

$$
\begin{aligned}
& y=0.061 x^{2}-0.739 x+3.188 \\
& y=0.058 x^{2}-0.698 x+2.99 \\
& y=0.065 x^{2}-0.791 x+3.26
\end{aligned}
$$

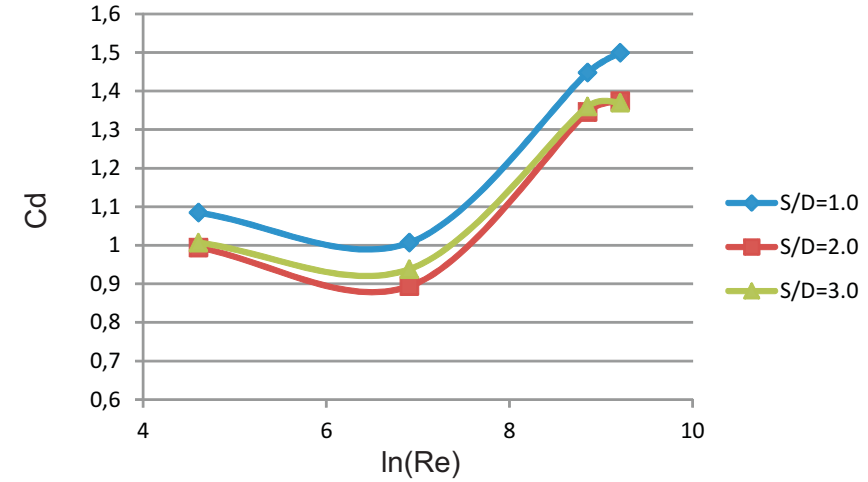

Fig. 3. Graphs of Tables II

We obtain the smallest drag coefficient of (9) when $R_{e}=$ 418.54. Equation (10) is obtained when $R_{e}=410.09$. Finally (11) is obtained when $R_{e}=443.87$.

\section{REFERENCES}

[1] S. Singha and K. Sinhamahapatra, "Flow past a circular cylinder between parallel walls at low Reynolds numbers," Ocean Engineering, vol. 37, no. 8-9, pp. 757-769, 2010.

[2] H. Sarvghad-Moghaddam, N. Nooredin, and B. Ghadiri-Dehkordi, "Numerical simulation of flow over two side-by-side circular cylinders," Journal of Hydrodynamics, Ser. B, vol. 23, no. 6, pp. 792-805, 2011.

[3] C. Imron, B. Widodo, and T. Yuwono, "Numerical simulation of fluid flow around circular and I-shape cylinder in a tandem configuration," Applied Mathematical Sciences, vol. 7, no. 114, pp. 5657-5666, 2013.

[4] D. Z. Noor, M.-J. Chern, and T.-L. Horng, "An immersed boundary method to solve fluid-solid interaction problems," Computational Mechanics, vol. 44, no. 4, pp. 447-453, 2009.

[5] A. L. E. Silva, A. Silveira-Neto, and J. Damasceno, "Numerical simulation of two-dimensional flows over a circular cylinder using the immersed boundary method," Journal of Computational Physics, vol. 189, no. 2, pp. 351-370, 2003.

where $y$ is $C_{d}$ and $x$ is $\ln \left(R_{e}\right)$. 